\title{
Parallel Streams for the Relay of Vibrissal Information through Thalamic Barreloids
}

\author{
Tiphaine Pierret, Philippe Lavallée, and Martin Deschênes \\ Centre de Recherche Université Laval-Robert Giffard, Hôpital Robert Giffard, Québec G1J 2G3, Canada
}

\begin{abstract}
This study investigated the organization of a vibrissal pathway that arises from the interpolar division of the spinal trigeminal complex (SP5i), transits through the ventral posterior medial nucleus (VPM), and innervates the somatosensory cortical areas in the rat. Using Fluoro-Gold and biotinylated dextran amine, respectively, as retrograde and anterograde tracers, the following organization plan was disclosed. The SP5i projection arises from a population of small-sized neurons that selectively innervate the ventral lateral part of VPM. In cytochrome oxidase-stained material, this region does not display any barreloid arrangement, but Fluoro-Gold injections in single barrel columns labeled rods of cells that extend caudally into the ventral lateral division of VPM. Thus, on the basis of retrograde labeling, barreloids were divided into core and tail compartments, which correspond to the rod segments running across the dorsal and ventral lateral parts of
\end{abstract}

On each side of the rat snout, there are five horizontal rows of whiskers that form an orderly array of low-threshold mechanoreceptors. Each peripheral fiber innervating these mechanoreceptors responds to only one vibrissa and, centrally, the arrangement of the vibrissal pad is maintained in arrays of cellular aggregates referred to as barrelettes (brainstem), barreloids (thalamus), and barrels (cortex). Brainstem nuclei that receive vibrissal primary afferents include the principal trigeminal nucleus (PR5) and all subdivisions of the spinal trigeminal complex (SP5). Each of these (sub)nuclei contributes axons to the trigeminothalamic tract, but the main stream of ascending fibers arises from the PR5 and the interpolar division of the SP5 (SP5i).

Anatomical studies provided clear evidence for a one-to-one relationship between single whiskers and corresponding modules in the PR5, in the ventral posterior medial nucleus (VPM), and in the barrel cortex (Chmielowska et al., 1989; Lu and Lin, 1993; Williams et al., 1994; Agmon et al., 1995; Veinante and Deschênes, 1999). The organization of the SP5i-thalamo-cortical axis, however, remains poorly understood. This axis comprises, at least, two pathways: one that projects to the posterior group nucleus (Po) and another to VPM (Chiaia et al., 1991a; Williams et al., 1994; Veinante et al., 2000). A central issue concerning latter projections bears on their relationships with the barreloid/barrel system. Traditionally, barreloids have been identified by either cytochrome oxidase $(\mathrm{CO})$ histochemistry or retrograde labeling after deposit of a tracer into a single barrel. Using horseradish peroxidase as a retrograde tracer, Saporta and Kruger (1977) first reported the rod-like clustering of VPM cells that project to the rat barrel cortex. Although injections were clearly not restricted to the size of a single barrel, VPM rods consisted of a sharply defined core of neurons in rostral VPM in which virtually all neurons were labeled; caudally, rods thinned out, forming a tail in which labeled and

Received May 26, 2000; revised July 18, 2000; accepted July 21, 2000

This work was supported by Medical Research Council of Canada Grant MT 5877. T.P. was supported by a CIEC Training Grant from the Government of Canada.

Correspondence should be addressed to Martin Deschênes, Centre de Recherche, Université Laval-Robert Giffard, Hôpital Robert-Giffard, 2601 de la Canardière, Québec G1J 2G3, Canada. E-mail: martind@microtec.net.

Copyright (C) 2000 Society for Neuroscience $0270-6474 / 00 / 207455-08 \$ 15.00 / 0$
VPM, respectively. Double-labeling experiments revealed that SP5i afferents innervate the tail of barreloids. The anterograde labeling of thalamocortical axons show that most "core cells" project to a single barrel column, whereas some "tail cells" give rise to branching axons that innervate the second somatosensory area and the dysgranular zone of the barrel field. Injections that straddled the transition zone between the core and tail regions disclosed cells projecting to a single barrel column and to the surrounding dysgranular zone. These results suggest that the projection of "barreloids cells" to the granular and/or dysgranular zones relates to the class of prethalamic input(s) they receive.

Key words: whiskers; barrels; ventral posterior medial nucleus; thalamocortical projections; trigeminothalamic afferents; spinal trigeminal nucleus unlabeled cells were intermingled. Similar rod-like clusters, although narrower in size, were evidenced in mice after tracer injections restricted to a single barrel column (Hoogland et al., 1987). The three-dimensional structure of barreloids was later examined by Land et al. (1995) using CO histochemistry. Barreloids were reported to form curved tapering cylinders, their long axis lying normal to the VPM/Po border. Ventrolaterally, as they approach the ventral posterior lateral nucleus (VPL), the tips of barreloids were seen to converge midway through the rostrocaudal extent of VPM. So defined, barreloids did not exhibit a caudal extension, a tail, as evidenced in retrograde-labeling experiments. The mismatch between both sets of data seems to occur in the ventrolateral and caudal aspects of VPM in which SP5i afferents project. The present series of experiments were undertaken to clarify the input-output organization of this region of the rat thalamus.

\section{MATERIALS AND METHODS}

Experiments were made in 65 adult rats (Sprague Dawley) in accordance with the federally prescribed and university animal care and use guidelines (Olfer et al., 1993). Surgery was performed under ketamine $(75 \mathrm{mg} / \mathrm{kg})$ plus xylazine $(5 \mathrm{mg} / \mathrm{kg})$ anesthesia, and rats were given analgesics (Anafen, $5 \mathrm{mg} / \mathrm{kg}$ ) before being returned to their cage. In different experiments, biotinylated dextran amine (BDA) (molecular weight, 10,000 kDa; Molecular Probes, Eugene, OR) or Fluoro-Gold (FG) (Fluorochrome Inc., Denver, $\mathrm{CO}$ ) were used alone or in combination as anterograde and retrograde tracers, respectively. The stereotaxic coordinates of the atlas of Paxinos and Watson (1986) were used to target the injections in SP5i or VPM, and the recording of vibrissae-evoked responses was routinely done to ascertain the correct placement of the injections.

All injections were made by iontophoresis by means of small-sized micropipettes $(5-30 \mu \mathrm{m})$. Biotinylated dextran amine $(2 \%$ in $0.5 \mathrm{M}$ potassium acetate) was ejected with positive current pulses of 300-1000 nA for periods ranging from 20 to $30 \mathrm{~min}$. Fluoro-Gold ( $2 \%$ in $0.1 \mathrm{M}$ cacodylate buffer, pH 7.0) was ejected with positive current pulses of 100-1000 nA for periods of $10-15 \mathrm{~min}$. After survival periods of 1-7 d, animals were perfused with saline followed by a fixative containing $4 \%$ paraformaldehyde and $0.5 \%$ glutaraldehyde in phosphate buffer $(0.1 \mathrm{M}, \mathrm{pH} 7.4)$. Brains were post-fixed in the same fixative for $2 \mathrm{hr}$, cryoprotected overnight in $30 \%$ sucrose, and cut at $50 \mu \mathrm{m}$ on a freezing microtome.

Except for the cases of double labeling, sections were usually processed for CO histochemistry (Wong-Riley, 1979) before revealing BDA or FG. Biotinylated dextran was revealed using the avidin-biotin-peroxidase complex (ABC kit; Vector Laboratories, Burlingame, CA) and the nickel-3,3'- 

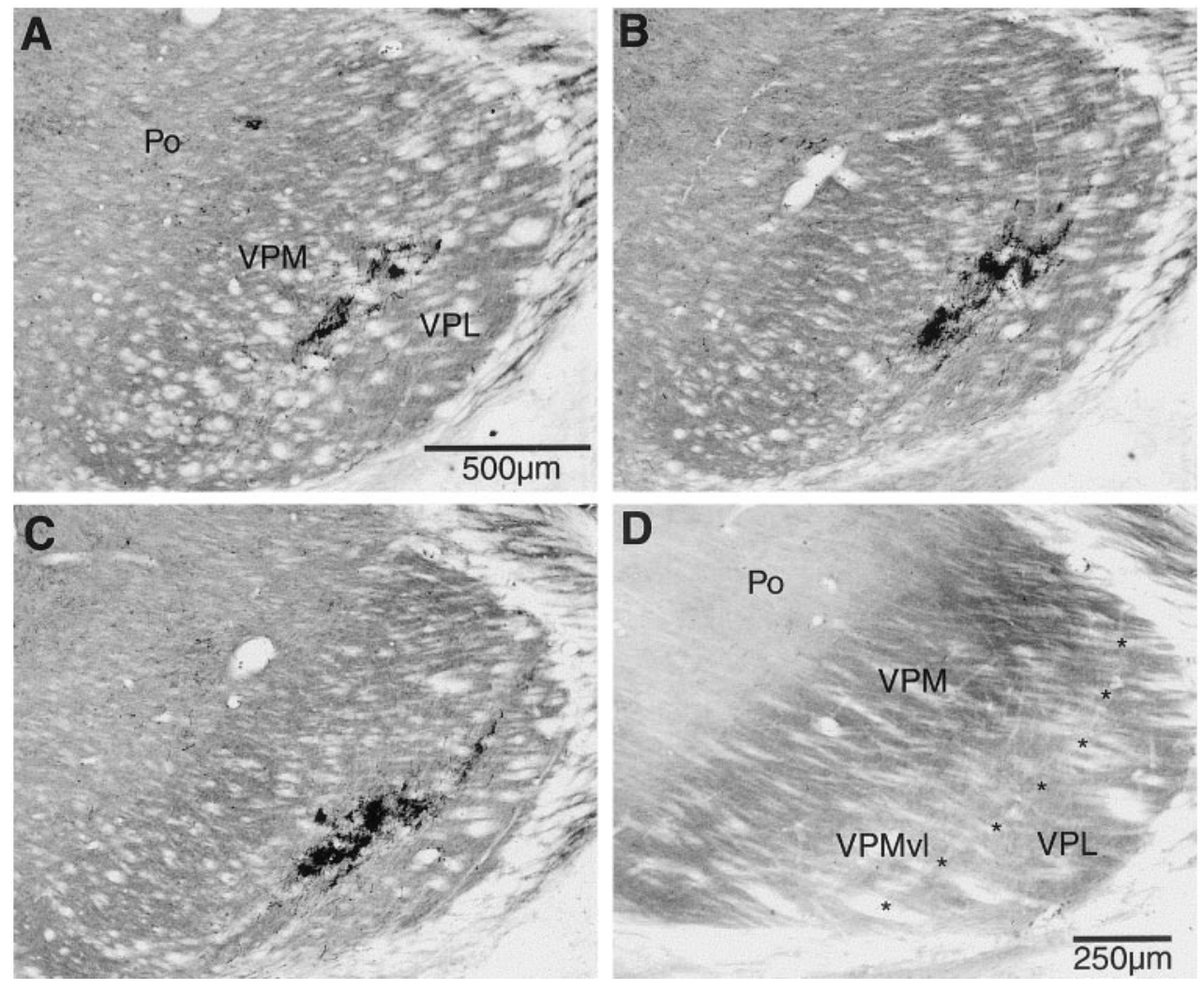

Figure 1. Distribution of SP5i terminal fields in the rat VPM. $A-C$ show, in COstained sections, the topography of BDAlabeled SP5i terminal fields at different frontal planes (rostral to caudal in $A-C$ ). Note the segregation of terminal fields in VPMvl. In tissue processed only for $\mathrm{CO}$ histochemistry $(D)$, VPMvl displays lighter staining than the dorsal part of the nucleus. Asterisks outline the frontier between VPM and VPL. Scale bar in $A$ also applies to $B$ and $C$.

diaminobenzidine tetrahydrochloride (DAB) substrate (Sigma, St. Louis, MO). Fluoro-Gold-labeled neurons were immunoreacted with an antiFluoro-Gold antiserum (Chemicon, Temecula, CA). After three rinses in PBS (0.01 M, pH 7.4) sections were incubated overnight in a solution containing 3\% normal goat serum (Vector Laboratories) $0.2 \%$ Triton $\mathrm{X}-100$, and anti-Fluoro-Gold antiserum $(1: 10,000)$. After three rinses in PBS, sections were incubated for $1 \mathrm{hr}$ in the secondary antibody (biotinylated goat IgG; Vector Laboratories), rinsed three times in PBS, and reacted with the ABC kit. After three rinses in Tris-buffered saline $(0.1 \mathrm{M}$, $\mathrm{pH}$ 7.6), peroxidase was revealed with the nickel-DAB substrate. In double-labeling experiments, FG was first revealed using a peroxidaselabeled secondary antibody (goat IgG; Chemicon) and DAB as a substrate (brown reaction product). Next, sections were processed for BDA histochemistry using the ABC kit and nickel-DAB (black reaction product). Finally, sections were mounted on gelatin-coated slides, dehydrated in alcohols, cleared in toluene, and coverslipped without counterstaining. Labeled material was drawn with a camera lucida using 25 or $40 \times$ objectives. Morphometric analysis and three-dimensional reconstructions were made with the Neurolucida and Neurotrace software (MicroBrightField Colchester, VT). The somatic cross-sectional areas of retrogradely labeled neurons were measured from tracings of the perimeters of labeled neurons drawn with the aid of a camera lucida using a $40 \times$ objective. The location of injection sites and projection foci was assessed from the nuclear divisions and cytoarchitectonic features outlined by the $\mathrm{CO}$ stain. In the present paper, the term "dysgranular zone" of S1 refers to the region in and around the barrel field in which layer 4 displays a low $\mathrm{CO}$ reactivity.

\section{RESULTS}

\section{The ventral lateral part of VPM receives SP5i afferents}

In Nissl-stained material, no cytoarchitectonic subdivision can be distinguished in the rat VPM. Neither calbindin nor parvalbumin immunoreactivity, which stain matrix and rod compartments in feline and primate VPM, reveal homologous territories in rodents. The topography of SP5i projections, however, clearly delineates a ventrolateral region to which we shall refer as the VPMvl. Figure $1 A-C$ shows a series of photomicrographs that illustrate the extent of this SP5i recipient zone. Delimited laterally by the VPL, VPMvl is a half-crescent-shaped region that approximately corresponds to the lower tier of VPM. It is thicker caudally and thins out rostrolaterally. In CO-stained material, VPMvl often displays a lighter coloration, which is better appreciated when the enzymatic reaction is allowed to fully develop (Fig. 1D). That V PMvl is distinct from the dorsal aspect of the nucleus is further indicated by the distri- bution of retrogradely labeled trigeminothalamic cells after FG injections restricted to either parts of the nucleus (Fig. 2). Injections in dorsal VPM, with minimal spread to VPMvl, produces heavy retrograde labeling in PR5 barrelettes but very sparse labeling in SP5i. Conversely, injections centered in VPMvl label neurons principally in SP5i and a smaller population of cells in the PR5.

\section{Small-sized SP5i neurons project to VPMvl}

In a previous study (Veinante et al., 2000), we reported that two types of SP5i fibers convey vibrissal information to the thalamus: thick, fast-conducting fibers that project to Po and thin, slowconducting axons that project to VPM. The two groups of axons were supposed to arise, respectively, from the large and small cells identified previously in SP5i by Phelan and Falls (1991). To test this hypothesis, we measured the cross-sectional area of SP5i cell bodies retrogradely labeled after FG deposits restricted to dorsal Po or V PMvl (Figs. 2B,C). The histograms of Figure 3 show that indeed the two populations of neurons differ in size. Cells projecting to Po exhibit larger somata than those projecting to V PMvl (mean \pm SD cross-sectional area, $396 \pm 153$ vs $153 \pm 64 \mu \mathrm{m}^{2}$ ). This difference is statistically significant (Student's $t$ test, $p<0.001$ ), thus confirming the distinct cellular origin of SP5i projections to V PMvl and Po.

\section{The core-tail structure of thalamic barreloids}

To determine whether VPMvl relay neurons project to the barrel cortex and whether they are part of thalamic barreloids, small FG injections were made in layer 4 of physiologically identified barrel columns. The use of fine micropipettes $(10 \mu \mathrm{m})$ and low-intensity currents (100-200 nA) reduced tracer diffusion to neighbor barrels, but spread to the supragranular layers over the injected barrel was unavoidable. Of 25 attempts to inject a single barrel column, eight cases were successful. These include barrel columns representing whiskers B2, B4, C2, C3, D2, D3, and E3. Fluoro-Gold injections restricted to a single barrel column label sharply defined arrays of cells in dorsal VPM (Fig. 4). Like the barreloids outlined by CO staining (Land et al., 1995), arrays are $\sim 100 \mu \mathrm{m}$ wide and extend from the VPM/Po border toward VPL. In some sections, 

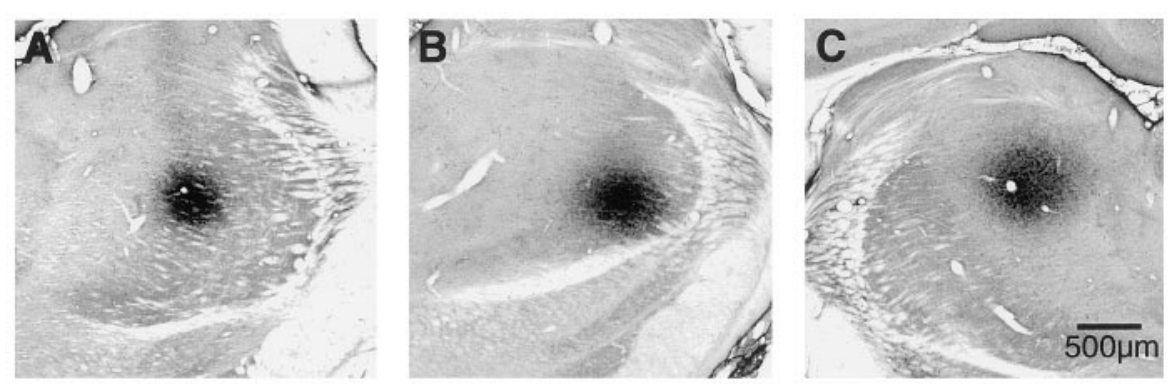

VPM dorsal
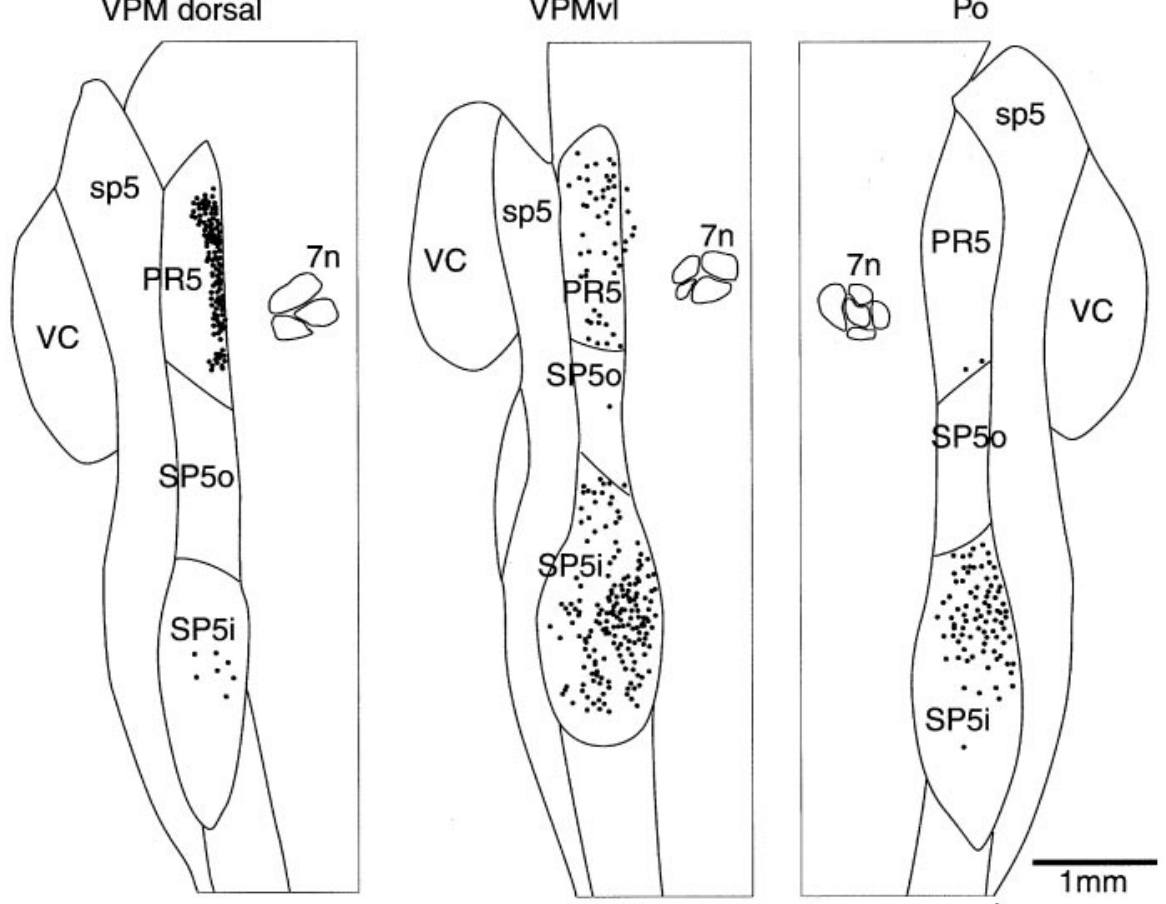

Figure 2. Distribution of retrogradely labeled trigeminothalamic cells after FG injections in different parts of the somatosensory thalamus. Injections sites in dorsal VPM, VPMvl, and Po are shown in $A-C$, with the corresponding maps of retrogradely labeled cells below. Each map represents the counts of five consecutive sections. sp5, Spinal trigeminal tract; $S P 5 o$, oral division of the spinal trigeminal complex; $V C$, ventral cochlear nucleus; $7 n$, tract of the facial nerve.

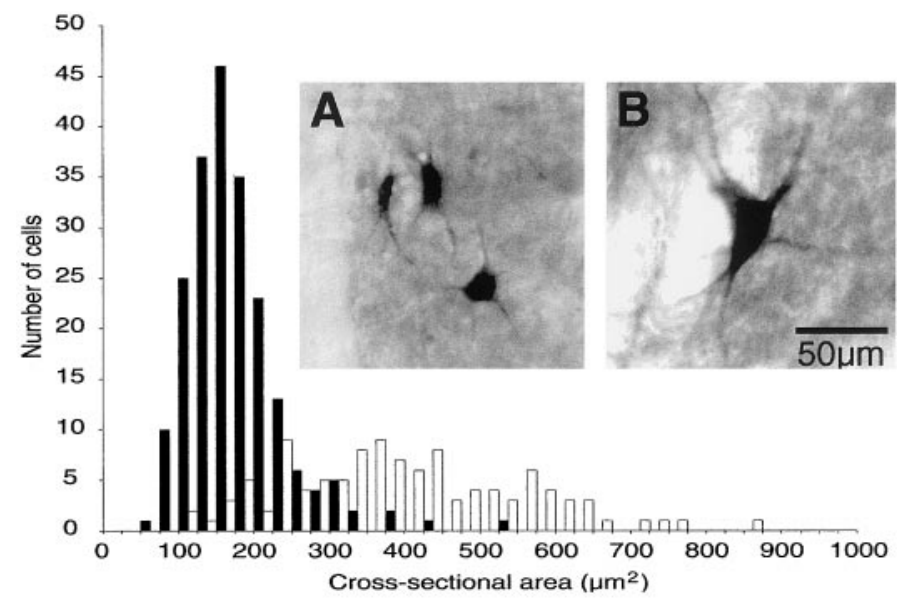

Figure 3. Comparative distributions of the cross-sectional areas of cell somata of SP5i neurons that project to VPMvl (black bars) and Po (white bars). Insets $A$ and $B$ show FG-labeled cells projecting to VPMvl and Po, respectively. Injection sites in Po and VPMvl are shown in Figure 2.

especially after the injection of barrel columns in C and D rows, $\mathrm{CO}$ light septa are seen flanking the rod of cells. These compact clusters of neurons form the core of barreloids. On reaching VP$\mathrm{Mvl}$, rods bend caudalward, forming a long string of cells in the posterior part of the nucleus (Fig. $4 C, F$ ). In this tail region, arrays differ from the rostral zone by displaying a lower cell density and less precise borders. The three-dimensional structure of barreloids B2 and D3 is shown in Figure 5 in which the core and tail regions are highlighted in different gray tones. It must be stressed that, in the absence of anterograde labeling, no sharp border can be drawn between the dorsal part of VPM and VPMvl. Thus, the transition between the core and tail of barreloids was assumed to occur where the rod of cells bent horizontally in the caudal direction. This transition occurs gradually in barreloids representing whiskers in D and $\mathrm{E}$ rows but manifests by a sharper bend in barreloids of the $\mathrm{A}$ and B rows (see sagittal representations in Fig. 5). Injections that spread to two adjacent barrel columns label cell clusters approximately twice as wide but with a similar three-dimensional structure. Thus, these results show that each barrel column receives input from an array of thalamic neurons which, in dorsal VPM and VPMvl, form the core and tail of barreloids, respectively.

\section{SP5i afferents innervate the tail of barreloids}

Direct evidence that SP5i afferents innervate the tail of barreloids was provided by double-labeling experiments (five cases) in which BDA and FG were injected, respectively, in SP5i and physiologically identified barrel columns. A clear overlap of anterograde axonal and retrograde cellular labeling was observed in two experiments. Figure 6 shows the distribution of SP5i axons with respect to the core and tail of barreloids $\mathrm{C} 2 / \mathrm{C} 3$. In the core region, no anterograde labeling is observed, although labeled fibers are present in VPMvl, beneath the cluster of stained somata (Fig. 6C). In sections passing through the tail of the same barreloids, however, numerous darkly stained fibers and terminations are seen among the brown-labeled somata (Fig. 6D). Failure to observe overlap in the other experiments likely relates to the small size of the SP5i injections, because in those cases projection foci were seen next to the clusters of retrogradely labeled cells. 

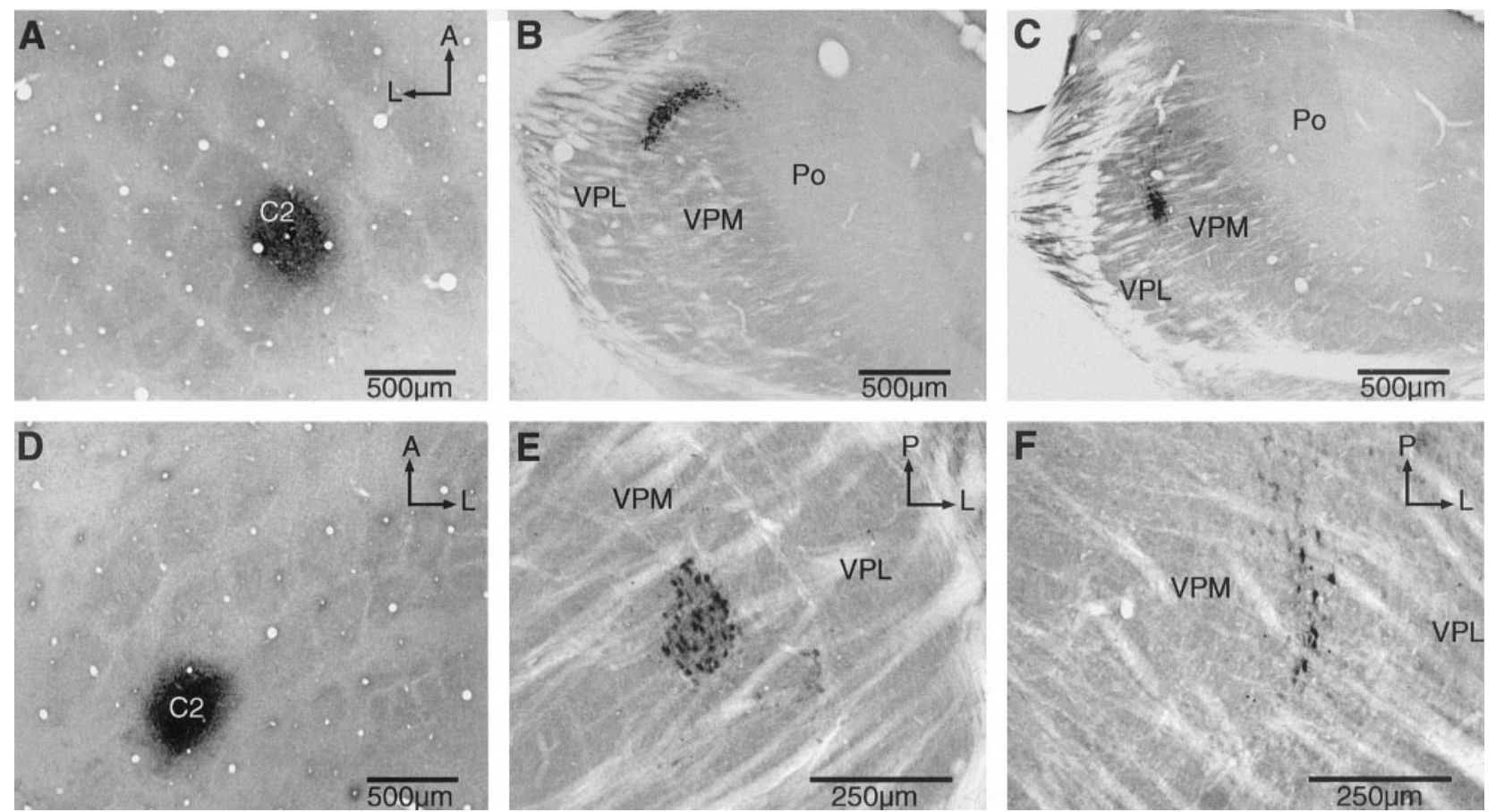

Figure 4. Retrograde labeling in the rat VPM after FG injections in barrel column C2. Tangential sections in $A$ and $D$ show the cortical injection sites. $B$ and $C$ show, respectively, the core and tail of barreloid $\mathrm{C} 2$ in coronally cut sections. The core and tail of the same barreloid, as they appear in horizontal sections, are shown in $E$ and $F$, respectively. $A$, Anterior; $L$, lateral; $P$, posterior.

Figure 5. Three-dimensional structure of thalamic barreloids as revealed by the retrograde transport of $\mathrm{FG}$ injected in barrel columns D3 $(A)$ and B2 $(B)$. Horizontal, frontal, and sagittal views are shown from left to right. The black and gray dots represent, respectively, neurons within the core and tail of barreloids. The transition between the core and tail was assumed to occur at the dorsal VPM/ VPMvl border. $A$, Anterior; $D$, dorsal; $M$, medial; $P$, posterior.
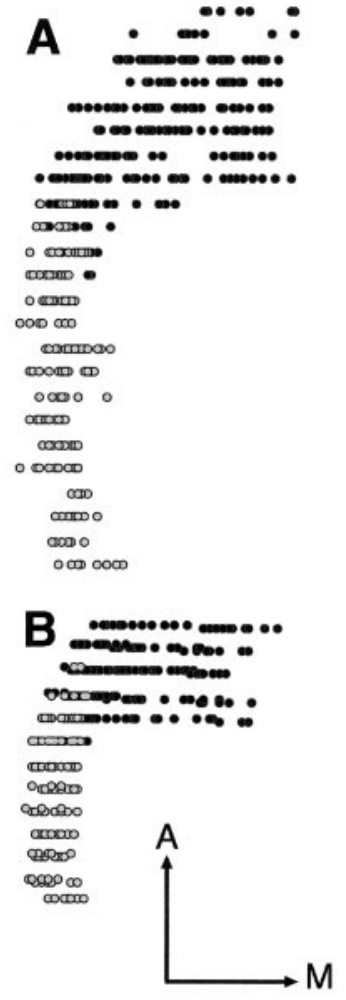

\section{Thalamocortical projections from the core and tail of barreloids}

The selective innervation of the tail of barreloids by SP5i afferents raises the issue of a differential projection from the core and tail subdivisions on the barrel cortex. This question was addressed by making BDA injections in dorsal VPM and VPMvl, respectively. As expected, injections in the dorsal VPM (four cases) label fibers
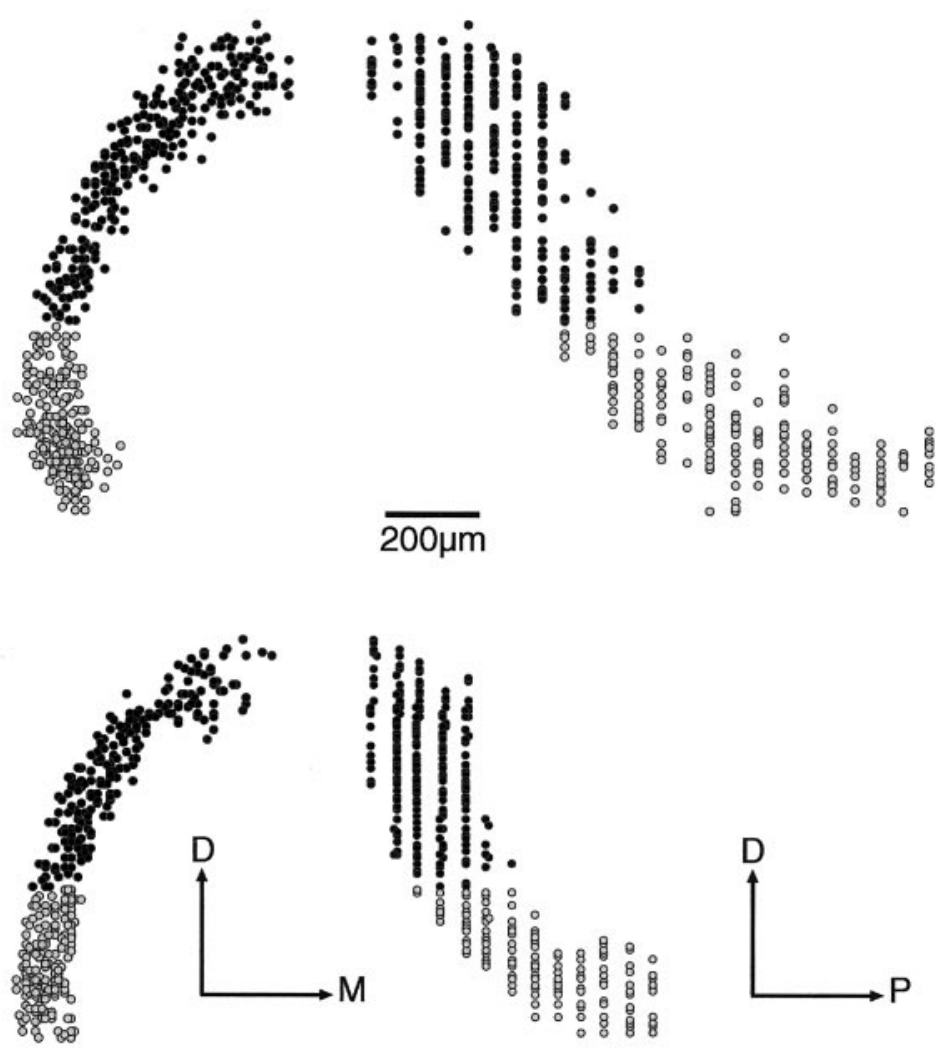

that project massively to the CO-reactive barrels and innervate more sparsely the upper layer 6 of the same barrel columns (Fig. $7 A, B)$. In contrast, injections restricted to the V PMvl (eight cases) highlighted projections across a broader expanse of the neocortex, which includes the second somatic sensory area (S2), the insular region, and the dysgranular zone of the primary somatic sensory area (S1) (Figs. $7 C, D, 8 B$ ). The most robust projection foci are 

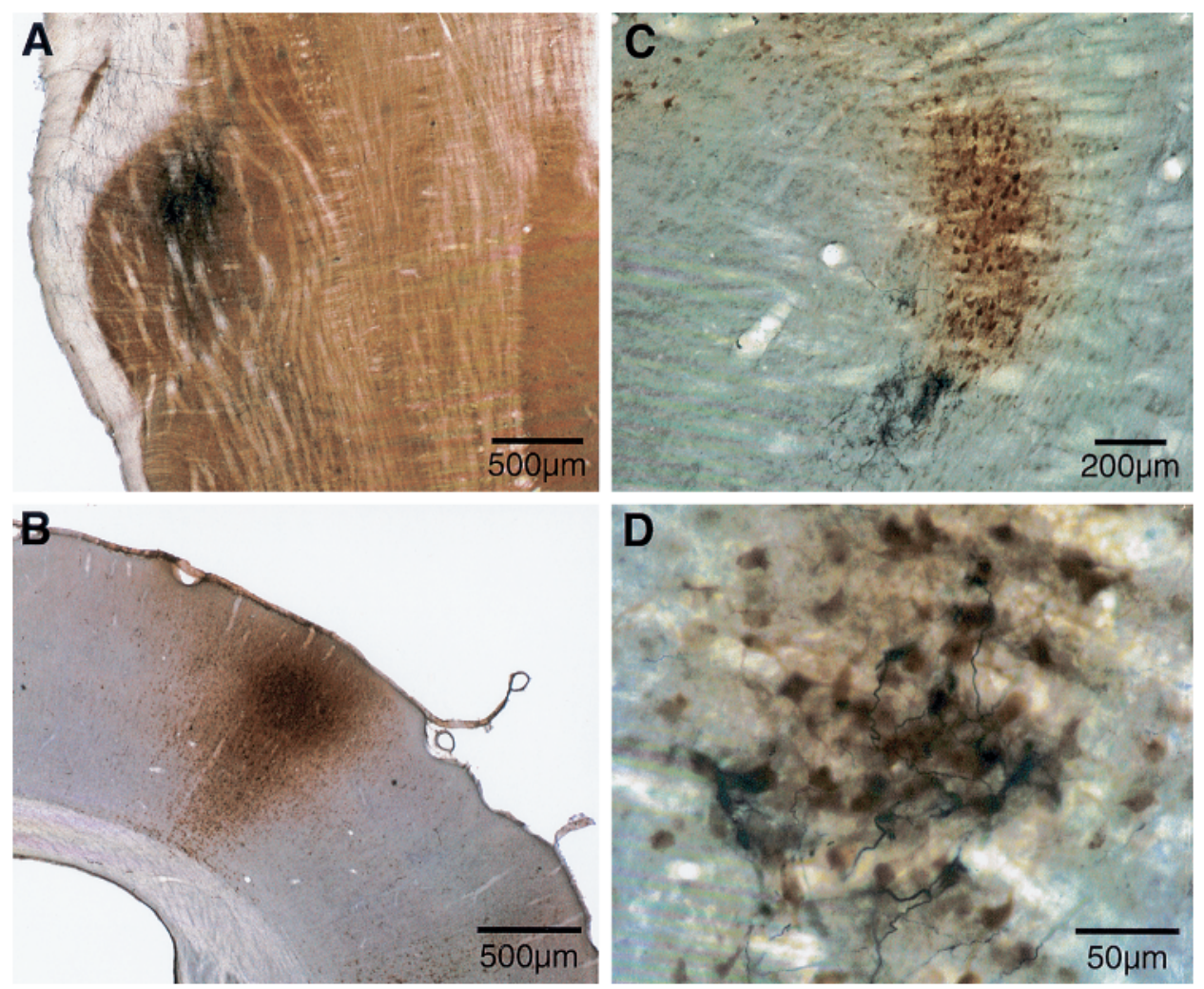

Figure 6. Selective innervation of the tail of barreloids by SP5i afferents. The injection sites of BDA in SP5i and of FG in barrel columns $\mathrm{C} 2 / \mathrm{C} 3$ are shown in $A$ and $B$, respectively. Dark blue SP5i fibers in $C$ are seen beneath the rod of brown-labeled somata forming the core of barreloids. In the tail region $(D)$, dark blue fibers and terminals are seen among the cluster of FG-labeled cells. observed in layers 4 and 6 of S2, whereas the concurrent anterograde labeling in S1 is principally concentrated in layers 3,4 , and 6 . Injections that straddled the transition zone between dorsal VPM and VPMvl (three cases) led to a mixed pattern of anterograde labeling consisting of dense projection foci in the barrel columns and a lighter projection to the surrounding dysgranular zone and $\mathrm{S} 2$.

Although injections of the size shown in Figure 7 label too many fibers to allow the complete reconstruction of but a few axonal arbors, at least three types of thalamocortical axons were clearly recognizable in our material. The "standard" type of thalamocortical axon that projects to a single barrel column was observed after injections restricted to dorsal VPM (Fig. $8 A$ ). By far, this type of axon seems to constitute the most prevalent thalamic input to the barrels. The second type of fiber consists of VPMvl axons that project to both S2 and the dysgranular zone of S1. The sparsity of these fibers in $\mathrm{S} 1$ allowed the complete reconstruction of 20 local axonal arbors which, in backward reconstructions, were all seen to stem from parent fibers that also projected to S2 (Fig. $8 C$ ). Injections that straddled the dorsal VPM/VPMvl border also provided clear evidence for a third type of fiber that innervates both a single barrel column and the surrounding dysgranular zone (Fig. 8D). This last type of fiber was intermingled with, and often hidden by, the numerous barrel-specific projecting axons. The one shown in Figure $8 D$ was sufficiently isolated from the rest of the projection focus to be fully reconstructed, but close examination of the material and several cases of partial reconstruction suggest that many axons of this type arise from the transition zone between dorsal VPM and VPMvl.

\section{DISCUSSION}

The present study provides evidence for a stream of vibrissal information that arises from a population of small-sized SP5i neurons, transits through the ventral lateral part of VPM, and innervates the barrel cortex and S2. This stream parallels the PR5-thalamo-cortical pathway, but differs from the latter on several aspects.

\section{The PR5 and SP5i projection systems}

In the vibrissal sensory system, first-order afferents have singlewhisker receptive fields and project on corresponding barrelettes in the PR5 and SP5i by means of axons that branch repeatedly throughout the trigeminal column (Hayashi, 1980, 1985; Jacquin et al., 1986b). The vast majority of PR5 cells that project to VPM have narrow, barrelette-bounded dendritic trees, which makes them responsive to the displacement of a single vibrissa (Shipley, 1974; Jacquin et al., 1988; Henderson and Jacquin, 1995; Veinante and Deschênes, 1999) In contrast, thalamic-projecting SP5i cells distribute dendrites across multiple barrelettes and thereby manifest a multi-whisker responsiveness (Woolston et al., 1982; Jacquin et al., 1986, 1989). The ascending SP5i multi-whisker stream is composed of at least two distinct subsystems: one that arises from small-sized neurons that project to V PMvl and another that arises from large multipolar neurons that project to Po (Veinante et al., 2000). For the sake of the present discussion, this latter subsystem shall not be considered further.

The whisker-like patterning of the terminal fields of PR5 axons in the rat VPM has been well documented at both the ensemble level and a single-cell level (Williams et al., 1994; Veinante and Deschênes, 1999). These fibers project principally to the dorsal part of the nucleus, forming bushy terminal fields whose size corresponds to that of the barreloids highlighted by CO staining (Williams et al., 1994; Land et al., 1995; Veinante et al., 1999). In VPMvl, the CO stain lightens, septal divisions disappear and, as a whole, the SP5i projection does not exhibit any clear whisker-like arrangement. Yet, single SP5i axons form narrow bushy arbors whose size and orientation suggest an intriguing continuity with the tip of the barreloids (Veinante et al., 2000).

A more global and comprehensive organizational plan emerges, however, when the thalamocortical projections to a single barrel column are examined by retrograde labeling. Each barrel column is seen to receive input from a rod of cells that extends across the PR5 and SP5i recipient zones of VPM. Thus, barreloids, as defined by $\mathrm{CO}$ staining, correspond to only the rostral segment of the rod traversing the dorsal VPM. We referred to this rostral segment as 

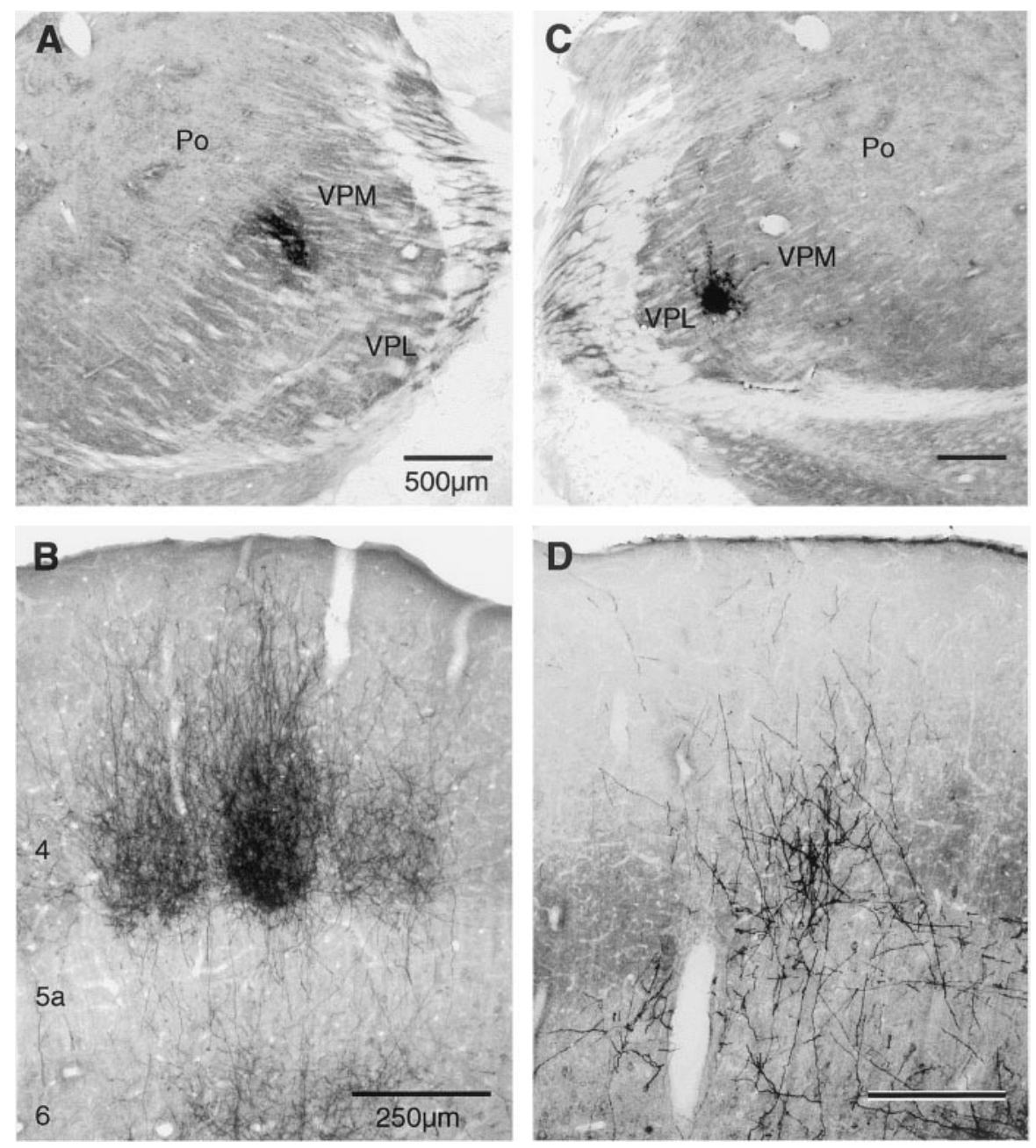

Figure 7. Thalamocortical projections arising from the dorsal part of VPM and from VPMvl. Biotinylated dextran injections in dorsal VPM $(A)$ label fibers that terminate in the barrels $(B)$, whereas injections in VPMvl $(C)$ produce terminal labeling in interbarrel regions $(D)$. Scale bars: $C, 500 \mu \mathrm{m} ; D, 250 \mu \mathrm{m}$.

the core of barreloids in Results. Cells within the core project only to $\mathrm{S} 1$, and the vast majority seem to innervate a single barrel column. The SP5i recipient segment contains the tail of barreloids, and the residing cells project massively to S2 and less densely to the dysgranular zone of S1. However, the distribution of axonal branches in S1 does not suggest any specific topographic relationship with single barrel columns. Therefore, cells with branching axons can hardly account for the barrel specificity of the projections arising from the tail of barreloids. More likely candidates are the cells that project to a single barrel column and to the surrounding dysgranular zone. Tracer injections suggest such cells to be located in the transition zone between dorsal VPM and VPMvl. For the moment, this zone remains ill-defined in regards to both the topographic distribution of trigeminal inputs and the dendroarchitecture of the relay cells. If one assumes dendritic field sizes of 250-300 $\mu \mathrm{m}$ for VPM neurons (Harris, 1987; Chiaia et al., 1991b), many cells straddling the VPM/VPMvl border would be in a position to receive convergent inputs from both PR5 and SP5i afferents. Indeed, ultrastructural and electrophysiological studies provided evidence for the convergence of PR5 and SP5i axons onto single VPM neurons (Chiaia et al., 1991a; Wang and Ohara, 1993; Freidberg et al., 1999), but the location of the cells contacted by both inputs remained undetermined. Intracellular recording of PR5- and SP5i-evoked synaptic responses and cell labeling will be required to settle this issue.

We know of no study that investigated, at a single-cell level, the diversity of thalamocortical projections arising from the rat VPM. Bulk anterograde-labeling studies suggest a rather homogeneous population of fibers that innervate single barrel columns (Chmielowska et al., 1989; Lu and Lin, 1993; Agmon et al., 1995), and the reconstructions of Jensen and Killackey (1987) provided several examples of such fibers that were labeled after horseradish peroxidase injections in the white matter beneath the barrel cortex. Interestingly, that study also disclosed other types of fibers, some resembling that illustrated in Figure $8 D$. Although additional experiments will be required to match fiber types with the location of their cells of origin within the barreloids, it is already clear that barrel columns receive input from more than one type of VPM fiber and that the thalamic neurons from the core and tail of barreloids innervate different zones of the somatosensory areas. Our anatomical data suggest that, whether a cell projects to a single barrel column, to a barrel column and the surrounding dysgranular zone, or again to the dysgranular zone and S2, relates to its location in the barreloids. Accordingly, cells on which PR5 and SP5i afferents converge might be the ones that innervate the granular and dysgranular zones of the barrel field.

\section{The requirement for parallel pathways}

To what extent SP5i projections contribute to the multi-whisker responses of cells in the barrel cortex is difficult to assess from previous physiological studies. It is well established that, in undrugged or lightly anesthetized rats, the receptive field of VPM cells is composed of multiple whiskers (Simons and Carvell, 1989; Armstrong-James and Callahan, 1991; Nicolelis and Chapin, 1994; Freidberg et al., 1999). A dominant whisker is always found; its deflection induces a robust short latency response, whereas the deflection of the other whiskers composing the field produces responses of lower magnitude at longer latency. The latter responses are abolished by SP5i lesion, which reduces the receptive field to one or two whiskers (Rhoades et al., 1987; Freidberg et al., 1999). Thus, under light anesthesia, one would expect cortical cells to exhibit similar response profiles after stimulation of the domi- 


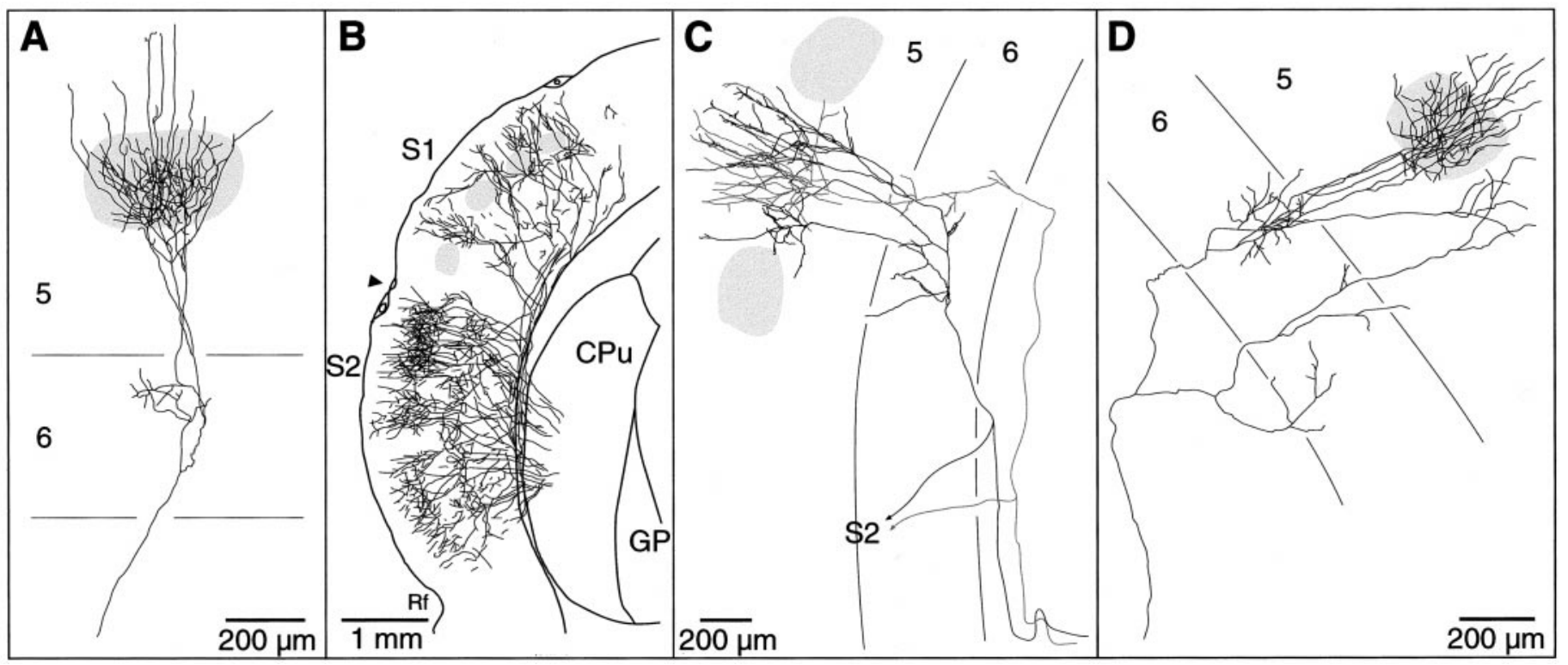

Figure 8. Camera lucida reconstructions of thalamocortical fibers arising from the dorsal part of VPM and from VPMvl. The "standard" type of barrel-specific fiber, which was labeled after BDA injections in dorsal VPM, is shown in $A$. B shows the areal distribution of thalamocortical axons labeled after BDA injection in VPMvl. Four consecutive sections were used for the reconstruction. Branching axons that project to the dysgranular zone of S1 and to S2 are shown in $C$. A fiber that innervates both a barrel and the surrounding dysgranular zone is shown in $D$ (see Results). Shaded patches represent cortical barrels. $C P u$, Caudate putamen; $G P$, globus pallidus; $R f$, rhinal fissure.

nant and nondominant whiskers. Indeed they do (ArmstrongJames et al., 1991). However, the SP5i-mediated multi-whisker responses of VPM neurons was found to bear no contribution to the evoked discharges of cells in cortical barrels, the responses to the nondominant vibrissae being mediated by intracortical rather than thalamocortical connections (Armstrong-James and Callahan, 1991). One must then conclude that the late-arriving multi-whisker thalamic input is in some way suppressed by an intracortical inhibitory mechanism. This seems plausible, considering that thalamocortical axons make a number of synaptic contacts with GABAergic neurons within the barrels (White, 1985; Keller, 1995). This explanation, however, leaves unresolved the contribution of SP5i afferents to the sensory processes taking place in the barrel cortex.

Insofar as PR5 and SP5i cells receive input from the same primary afferents, the requirement for parallel vibrissal pathways likely relates not to the processing of different sensory submodalities but to complexities arising from the operation of a mobile sensory organ. Rats commonly explore their environment and palpate objects by repetitive forward and backward sweeping movements of the mystacial vibrissae. Thus, parallel processes are required for gating sensory signals induced by self-initiated movements, for filtering redundant information, and for deblurring "vibrissal images" acquired during head and body displacements. Whether the SP5i network fulfills any of these functions is currently unknown, but in view of the apparent idleness of this system in head-restrained animals, one is inclined to believe that the type of information conveyed to the cortex by this subsystem relates to the processing of vibrissal information during active whisking.

\section{REFERENCES}

Agmon A, Yang LT, Jones EG, O’Dowd DK (1995) Topological precision in the thalamic projection to neonatal mouse barrel cortex. J Neurosci 15:549-561.

Armstrong-James M, Callahan CA (1991) Thalamocortical processing of vibrissal information in the rat. II. Spatiotemporal convergence in the thalamic ventroposterior medial nucleus $(\mathrm{VPm})$ and its relevance to generation of receptive fields of S1 cortical barrel neurones. J Comp Neurol 303:211-224.

Armstrong-James M, Callahan CA, Friedman MA (1991) Thalamocortical processing of vibrissal information in the rat. I. Intracortical origins of surround but not center-receptive fields of layer IV neurones in the rat S1 barrel field cortex. J Comp Neurol 303:193-210.

Chiaia NL, Rhoades RW, Bennett-Clarke CA, Fish S, Killackey HP (1991a) Thalamic processing of vibrissal information in the rat. I. Af- ferent input to the ventral posterior medial and posterior nuclei. J Comp Neurol 314:201-216.

Chiaia NL, Rhoades RW, Fish SE, Killackey HP (1991b) Thalamic processing of vibrissal information in the rat. II. Morphological and functional properties of ventral posterior medial nucleus and posterior nucleus neurons. J Comp Neurol 314:217-236.

Chmielowska J, Carvell GE, Simons DJ (1989) Spatial organization of thalamocortical and corticothalamic projection systems in the rat SmI barrel cortex. J Comp Neurol 285:325-338.

Freidberg MH, Lee SM, Ebner FF (1999) Modulation of receptive field properties of thalamic somatosensory neurons by the depth of anesthesia. J Neurophysiol 81:2243-2252.

Harris RM (1987) Morphology of physiologically identified thalamocortical relay neurons in the rat ventrobasal thalamus. J Comp Neurol 251:491-505.

Hayashi H (1980) Distribution of vibrissae afferent fiber collaterals in the trigeminal nuclei as revealed by intra-axonal injection of horseradish peroxidase. Brain Res 183:442-446.

Hayashi H (1985) Morphology and central terminations of intra-axonally stained large myelinated primary afferent fibers from facial skin in the rat. J Comp Neurol 237:195-215.

Henderson TA, Jacquin MF (1995) What makes subcortical barrels? In: Cereb cortex, Vol 12, The barrel cortex of rodents (Jones EG, Diamond IT, eds), pp 123-187. New York: Plenum.

Hoogland PV, Welker E, Van der Loos H (1987) Organization of the projections from barrel cortex to thalamus in mice studied with Phaseolus vulgaris-leucoagglutinin and HRP. Exp Brain Res 68:73-87.

Jacquin MF, Mooney RD, Rhoades RW (1986) Morphology, response properties, and collateral projections of trigeminothalamic neurons in brainstem subnucleus interpolaris of rat. Exp Brain Res 61:457-468.

Jacquin MF, Golden JP, Panneton WM (1988) Structure and function of barrel "precursor" cells in trigeminal nucleus principalis. Dev Brain Res 43:309-314.

Jacquin MF, Barcia M, Rhoades RW (1989) Structure-function relationships in rat brainstem subnucleus interpolaris. IV. Projection neurons. J Comp Neurol 282:45-62.

Jensen KF, Killackey HP (1987) Terminal arbors of axons projecting to the somatosensory cortex of the adult rat. I. The normal morphology of specific thalamocortical afferents. J Neurosci 7:3529-3543.

Keller A (1995) Synaptic organization of the barrel cortex. In: Cereb cortex, Vol 12, The barrel cortex of rodents (Jones EG, Diamond IT, eds), pp 221-262. New York: Plenum.

Land PW, Buffer SA, Yaskosky DJ (1995) Barreloids in adult rat thalamus: three-dimensional architecture and relationship to somatosensory cortical barrels. J Comp Neurol 355:573-588.

Lu S-M, Lin RC-S (1993) Thalamic afferents of the rat barrel cortex: a light- and electron-microscopic study using Phaseolus vulgaris leucoagglutinin as an anterograde tracer. Somatosens Mot Res 10:1-16.

Nicolelis MAL, Chapin JK (1994) Spatiotemporal structure of somatosensory responses of many neurons ensembles in the rat ventral posterior medial nucleus of the thalamus. J Neurosci 14:3511-3542. 
Olfer ED, Cross BM, McWilliams AA (1993) Guide to the care and use of experimental animals. Bradda, Canada: Canadian Council on Animal Care.

Paxinos G, Watson C (1986) The rat brain in stereotaxic coordinates, Ed 2. Sydney: Academic.

Phelan KD, Falls WM (1991) A comparison of the distribution and morphology of thalamic, cerebellar and spinal projection neurons in rat trigeminal nucleus interpolaris. Neuroscience 40:497-511.

Rhoades RW, Belford GR, Killackey HP (1987) Receptive-field properties of rat ventral posterior medial neurons before and after selective kainic acid lesions of the trigeminal brain stem complex. J Neurophysiol 57:1577-1600.

Saporta S, Kruger L (1977) The organization of thalamocortical relay neurons in the rat ventrobasal complex studied by the retrograde transport of horseradish peroxidase. J Comp Neurol 174:187-208.

Shipley MT (1974) Response characteristics of single units in the rat's trigeminal nuclei to vibrissa displacements. J Neurophysiol 37:73-90.

Simons DJ, Carvell GE (1989) Thalamocortical response transformation in the rat vibrissa-barrel system. J Neurophysiol 61:311-330.

Veinante P, Deschênes M (1999) Single- and multi-whisker channels in the ascending projections from the principal trigeminal nucleus in the rat. J Neurosci 19:5085-5095.

Veinante P, Jacquin MF, Deschênes M (2000) Thalamic projections from the whisker-sensitive regions of the spinal trigeminal complex in the rat. J Comp Neurol 420:233-243.

Wang B-R, Ohara PT (1993) Convergent projections of trigeminal afferents from the principal nucleus and subnucleus interpolaris upon rat ventral posteromedial thalamic neurons. Brain Res 629:253-259.

White EL (1985) Terminations of thalamic afferents. In: Cereb cortex, Vol 5, Sensory-motor areas and aspects of cortical connectivity (Jones EG, Peters AJ, eds), pp 271-289. New York: Plenum.

Williams MN, Zahm DS, Jacquin MF (1994) Differential foci and synaptic organization of the principal and spinal trigeminal projections to the thalamus in the rat. Eur J Neurosci 6:429-453.

Wong-Riley MTT (1979) Changes in the visual system of monocularly sutured or enucleated cats demonstrable with cytochrome oxidase histochemistry. Brain Res 171:11-28.

Woolston DC, LaLonde JR, Gilson JM (1982) Comparison of response properties of cerebellar and thalamic-projecting interpolaris neurons. J Neurophysiol 48:160-173. 